Bond University

Research Repository

\title{
Beer goggles: Blood alcohol concentration in relation to attractiveness ratings for unfamiliar opposite sex faces in naturalistic settings
}

Lyvers, Michael; Cholakians, Emma; Puorro, Megan; Sundram, Shanti

Published in:

Journal of Social Psychology

DOI:

$10.1080 / 00224540903366776$

Licence:

CC BY-NC-ND

Link to output in Bond University research repository.

Recommended citation(APA):

Lyvers, M., Cholakians, E., Puorro, M., \& Sundram, S. (2011). Beer goggles: Blood alcohol concentration in relation to attractiveness ratings for unfamiliar opposite sex faces in naturalistic settings. Journal of Social Psychology, 151(1), 105-112. https://doi.org/10.1080/00224540903366776

\section{General rights}

Copyright and moral rights for the publications made accessible in the public portal are retained by the authors and/or other copyright owners and it is a condition of accessing publications that users recognise and abide by the legal requirements associated with these rights.

For more information, or if you believe that this document breaches copyright, please contact the Bond University research repository coordinator. 
Beer Goggles: Blood Alcohol Concentration in Relation to Attractiveness Ratings for

Unfamiliar Opposite Sex Faces in Naturalistic Settings

Michael Lyvers, Ph.D.

Emma Cholakians, Postgraduate Diploma of Psychology

Megan Puorro, Postgraduate Diploma of Psychology

Shanti Sundram, Postgraduate Diploma of Psychology

Department of Psychology

Bond University

Gold Coast Qld 4229 Australia

Corresponding Author: Michael Lyvers

Phone: (61) (75) 5952565

Fax: (61) (75) 5952540

Email: $\underline{\text { mlyvers@staff.bond.edu.au }}$ 


\begin{abstract}
The popular notion that alcohol intoxication enhances perceptions of the physical attractiveness of the opposite sex has been inconsistently supported. The current study tested intoxicated and non-intoxicated persons of both genders in naturalistic settings after measuring their blood alcohol concentration (BAC) by a breath test. A sample of 80 heterosexual university student social drinkers was recruited at a campus pub and campus parties over a 3 month period to take a survey rating the attractiveness of unfamiliar faces of the opposite gender presented in photographs. Attractiveness ratings were positively correlated with BAC. Analysis of covariance (ANCOVA) was conducted on attractiveness ratings with independent variables of gender and BAC group, with three levels of the latter: non-intoxicated $(\mathrm{BAC}=0)$, moderately intoxicated (BAC .01\%-.09\%), and highly intoxicated (BAC .10\%-.19\%). Both intoxicated groups gave significantly higher attractiveness ratings than nonintoxicated controls. The findings confirm the "beer goggles" phenomenon of folk psychology for both genders, although the mechanism remains unclear.
\end{abstract}

Key words: alcohol, attraction, health \& safety 
Alcohol intoxication is often said to alter perception in such a way that potential sexual partners seem more attractive. This is reflected in popular phrases such as "beer goggles," "turn bow to wow," or "all the girls get prettier at closing time," which are humorous ways of referring to the situation where an intoxicated individual makes sexual advances that are later regretted in a sober state. Pennebaker (1979) reported that attractiveness ratings did in fact increase among both males and females in a bar setting as closing time approached. This was interpreted as an effect of increasing levels of blood alcohol, though the latter was not measured. Gladue and Delaney (1990) reported similar findings but again did not measure blood alcohol concentration (BAC). Kruse and Fromme (2005) administered placebo or alcoholic drinks to 80 males aged 21-30 years who said they drank at least 5 alcoholic drinks per occasion on at least one day per week over the previous 3 months, and asked them to rate photos of female models for desirability and to indicate the likelihood of risky sex with each. BAC readings were taken in both conditions, with false feedback given to participants in the placebo condition. As expected the more attractive models elicited higher ratings of desirability and stronger intentions to have risky sex. However, alcohol did not significantly affect perceptions of attractiveness or the likelihood of risky sex, though alcohol did influence the intent to discuss risks prior to sexual activity. The negative results may reflect the BAC levels achieved for these highly experienced drinkers, as the authors themselves acknowledged. Participants' alcohol tolerance may have prevented BACs of .05\%-.06\% from causing sufficient intoxication, as further suggested by the lack of significant difference between placebo and alcohol conditions in self-reported intoxication level. Jones et al. (2003) obtained attractiveness ratings for unfamiliar faces of both genders from 80 undergraduates of both genders across a range (1-6 UK units, where $1 \mathrm{UK}$ unit $=8 \mathrm{gm}$ 
alcohol) of alcohol consumption in a campus pub. However, alcohol consumption was assessed by self-report only; BAC was not measured. Participants rated the attractiveness of faces shown to them on a computer screen. Results showed the expected enhancement effect of alcohol consumption on attractiveness ratings for opposite sex faces but not for same sex faces. Jones et al. suggested that alcohol consumption can increase the likelihood of risky sex by altering perceptions of attractiveness. Previous research has indicated that physical attractiveness can influence the likelihood of risky sexual behavior. Persons rated as highly attractive tend to elicit greater self-reported intentions of engaging in risky unprotected sex (Agocha \& Cooper, 1999; Kruse \& Fromme, 2005). Thus alcohol consumption may increase the likelihood of risky sex by increasing the perceived attractiveness of prospective sexual partners, in addition to impairing judgment.

How might alcohol intoxication increase perceived attractiveness of potential sex partners? Recent neurophysiological evidence indicates that alcohol acutely stimulates dopamine release from the mesolimbic dopamine reward system, an effect that is most evident at moderate doses (Olive \& Griffin, 2008). In laboratory animals, dopamine release mediates the increase in sex drive triggered by sex hormones (Putnam, Du, \& Hull, 2001), and drugs with strong pro-dopaminergic effects (such as cocaine, methamphetamine, and 1-dopa) are reported to increase sexual desire in humans (e.g., Rosen \& Ashton, 1993). Thus alcohol may increase sexual desire via a dopaminergic mechanism, accounting for the first part of Shakespeare's famous observation that alcohol "provokes desire but takes away the performance." On the other hand, mere exposure to alcohol-related cues has also been reported to increase attractiveness ratings, particularly in those who believe that alcohol stimulates sexual desire (Friedman, McCarthy, Forster \& Denzler, 2005). As alcohol intoxication is 
obviously an alcohol-related cue, disentangling the contributions of pharmacology from those of expectancy may prove difficult where alcohol-related increases in attractiveness ratings are concerned. The notion that "alcohol provokes the desire" has been around a long time and presumably such expectancies arose from experiences and observations. Friedman et al. suggested that exposure to alcohol-related cues implicitly activated participants' expectancies about how alcohol affects desire; the authors noted that the men in their study varied in such expectancies, and that such variation may have resulted from individual experiences with the pharmacological actions of alcohol. That is, among those who repeatedly experienced increases in sexual desire while on alcohol, a conditioned association of alcohol with sexual desire developed which was then elicited by the alcohol-related cues in the study. Friedman et al. thus leave open the possibility that alcohol has a pharmacological action promoting sexual desire (at least in some), and that such actions are the ultimate basis of common beliefs that alcohol has aphrodisiac properties.

The present study investigated the relationship between BAC and facial attractiveness ratings by heterosexual university student social drinkers of both genders in naturalistic settings (campus pub and campus parties). BAC was expected to be positively correlated with ratings of attractiveness for unfamiliar faces of the opposite gender in both men and women, consistent with the "beer goggles" phenomenon.

\section{Method}

\section{Participants}

A sample of 80 Bond University students was recruited from a campus pub and from campus parties over a 3 month period. The sample consisted of 41 single heterosexual men and 39 single heterosexual women, all between the ages of 18 (legal 
drinking age in Australia) and $29(M=24.06$ years, $S D=2.79)$ and all of whom reported that they at least occasionally consumed alcoholic beverages. No incentive was offered. The exclusion criteria were: failure to sign the informed consent and disclaimer form (see below), self reporting other than heterosexuality, identifying any of the stimulus photographs as familiar, not being in the 18-29 age range, having a BAC of more than $.19 \%$, or reporting that they never consume alcoholic beverages. Five prospective participants exceeded $.19 \%$ BAC and were thus excluded from the study due to ethical considerations. The protocol for this study was approved by the Bond University Human Research Ethics Committee prior to data collection.

\section{Materials}

Breathalyzer. A newly calibrated Alcolizer $\mathrm{HH}-1 / \mathrm{HH}-2$ (Alcolizer

Technology, 2007) breathalyzer was used to measure BAC. The breathalyzer was recalibrated in the middle of the data gathering period of 3 months to ensure accuracy.

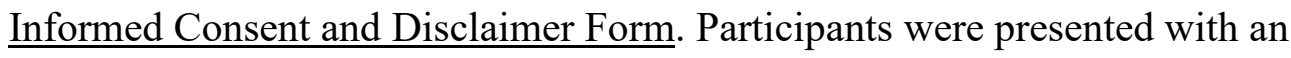
informed consent form that described the study as an investigation of factors influencing attractiveness, a statement indicating that their participation was entirely voluntary and anonymous, and a disclaimer relieving the researchers of any responsibility for the intoxicated persons' actions after the breath test was given.

Facial Attractiveness Surveys. Two surveys were constructed, one for heterosexual men and one for heterosexual women. Each survey contained 15 black and white headshot photographs with shoulders and hair visible. A master book with a laminated version of all the images in color was presented as well. On each page of each survey there were two $7 \times 8 \mathrm{~cm}$ photos per page, with the order of images randomized. No extreme or unusual features were shown in the images, nor excessive ornaments or make-up. The background was identical in each picture. The photos 
were obtained from a pilot study. Photos from www.lavalife.com, a public dating website in the United States, were chosen to ensure their unfamiliarity to participants. In the pilot study the photos were evaluated by a sober group of 40 undergraduate students who were instructed to rate the images on overall facial attractiveness. Thirty images of moderate attractiveness (15 of men, 15 of women) were chosen as the appropriate target stimuli for the present study. By not choosing photos with extremely high or low attractiveness ratings, potential floor or ceiling effects were avoided. The persons in the photos were all aged 20-28 years $(M=24.6$ years), similar to the participants. A 10 point Likert scale was located $2 \mathrm{~cm}$ below each photograph, with $1=$ very unattractive, $5=$ moderately attractive, and $10=$ very attractive.

Barratt Impulsiveness Scale (BIS-11) (Patton, Stanford \& Barratt, 1995). The BIS-11 is a self-report questionnaire designed to assess assess general impulsiveness as well as subtypes of impulsiveness. There are subscales measuring attentional impulsiveness, motor impulsiveness, and non-planning impulsiveness. All items are measured on a four-point Likert scale $(1=$ Rarely/Never, $2=$ Occasionally, $3=$ Often, $4=$ Almost Always/Always). Some items are reverse scored to avoid a response bias. The BIS-11 has been shown to have good psychometric properties according to Patton et al. and is a widely used index of trait impulsivity.

\section{Procedure}

With permission of the manager of the local pub, the surveys were distributed on the premises during the busy period between 9 p.m. and 12 a.m. on multiple nights over a 3 month period. Surveys conducted at campus parties were also administered between 9 p.m. and 12 a.m. over the same period. Prospective participants were approached, given a brief description of the experiment and asked if they were willing 
to participate in the study. The researcher then established if they met the inclusion criteria of being heterosexual, 18-29 years of age, and at least occasional consumers of alcoholic beverages. If so, they then signed the informed consent and disclaimer form. Participants were required to not have any alcoholic beverage for at least 10 minutes prior to testing (this was monitored by the researchers). Just before the breath test, participants were asked to rinse out their mouth with water (provided by the researchers) to ensure there was no alcohol residue that would inflate the BAC reading. The Alcolizer $H H-1 / H H-2$ was then used to measure BAC. A medium to hard blow for a steady 6 seconds was required. The disposable mouthpiece was then discarded and replaced by a new one, and the breathalyzer was reset for the next participant.

After the breath test the Facial Attractiveness Survey was administered. The master book was also presented for participants to view a color version of each photo. A writing utensil was provided. Participants were asked to rate the unfamiliar photographic facial images of opposite sex persons their own age on the 10-point Likert scale as indicated on the survey. Men were presented with facial photographs of women for attractiveness ratings, and women were presented with facial photographs of men for attractiveness ratings. The survey was completed out of view of the researchers. The BIS-11 followed the Facial Attractiveness Survey. Upon completion the questionnaires were put in an envelope with the breathalyzer rating for that participant written on the outside. No identifying information was contained with the questionnaires other than age and gender. The participant was thanked and given back their beverage if applicable. The current study was done in conjunction with another study using additional questionnaire materials; results for the other study will 
be reported elsewhere. Completion of all questionnaires for both studies took each participant 10-15 minutes in total.

\section{Results}

Initial correlational analysis revealed that, as predicted, attractiveness ratings were significantly positively correlated with $\mathrm{BAC}, r(80)=.234, p=.037$. In order to determine whether this relationship varied according to gender, participants were separated into three nearly equal groups based on BAC: non-intoxicated controls (these participants were not teetotalers but had either just arrived at the venue or were “designated drivers;" $\mathrm{BAC}=0, n=27)$, moderately intoxicated $(\mathrm{BAC}=.01 \%$ to $.09 \%, M=.046 \%, S D=.025 \% ; n=27)$ and highly intoxicated $(\mathrm{BAC}=.10 \%$ to $.19 \%$, $M=.156 \%, S D=.046 \% ; n=26)$. The BAC cutoff used to differentiate the moderately and severely intoxicated groups was chosen to provide nearly equal sample sizes for analysis of covariance (ANCOVA) as well as on grounds of face validity. Groups did not significantly differ on age or gender composition and contained nearly equal numbers of men and women. A 3 (BAC group) X 2 (gender) ANCOVA with total BIS-11 impulsivity score as the covariate (in order to control for the possible contribution of trait impulsivity to both BAC and attractiveness ratings) revealed a significant main effect of BAC group on attractiveness ratings, $F(2,72)=$ $6.41, p=.003$, observed power $=.89$. Tukey post-test showed that both the moderately intoxicated group $(M=4.67, S D=1.57)$ and the highly intoxicated group $(M=4.50, S D=1.47)$ gave significantly higher attractiveness ratings than did the non-intoxicated control group $(M=3.26, S D=1.70), \mathrm{p}<.01$. There was no effect of gender and no interaction.

Although total BIS-11 impulsivity scores were not correlated with BAC or attractiveness ratings, of the three BIS-11 subscales, non-planning impulsiveness was 
correlated with BAC, $r(79)=.23, p<.05$, but not attractiveness ratings, $r(79)=.14$, n.s.

\section{Discussion}

Consistent with predictions, the present results indicated that perceived attractiveness of unfamiliar opposite sex faces was positively associated with alcohol intoxication, confirming the "beer goggles" phenomenon. In contrast to these findings, Kruse and Fromme (2005) reported that although high levels of attractiveness increased the intent to engage in risky sex, alcohol had no effect on perceptions of a potential partner or the perceived likelihood of risky sex in heterosexual men. However, Kruse and Fromme's study assessed highly experienced regular drinkers at BACs that may have been insufficient to induce "beer goggles" given their presumed high level of alcohol tolerance. Other studies (e.g., Jones et al., 2003; Pennebaker, 1979) have reported evidence of such an effect, though BAC was not measured.

The relationship between BAC and attractiveness ratings did not differ by gender. Similar cognitive and perceptual effects of alcohol, including "beer goggles," are presumably likely in both genders at equivalent BACs. In partner selection physical attractiveness, and particularly facial attractiveness, is an important factor for both men and women (Bixler, 1989), thus "beer goggles" may plausibly have similar behavioral consequences for both genders in real-life situations.

A limitation of the present study is that other variables could conceivably mediate the relationship between BAC and attractiveness ratings. Personality is one such factor. For example, the trait of impulsiveness/reward sensitivity might plausibly be associated with both increased drinking and increased attractiveness ratings, as impulsivity has been linked to alcohol consumption (Dawe \& Loxton, 2004; Franken 
$\&$ Muris, 2006). This was the rationale for including total BIS-11 score as the covariate in the ANCOVA comparing BAC groups on attractiveness ratings.

Although one of the BIS-11 subscales - non-planning impulsiveness - was positively related to BAC, no BIS-11 measure was related to attractiveness ratings. This negative result helps strengthen the notion of a direct relationship between BAC and attractiveness ratings but of course does not rule out the possible contributions of other personality factors. A further plausible interpretation is that both BAC and sexual desire may have increased over time at the pub or party, another third variable effect. Perhaps pub patrons and partiers simply became both more intoxicated and more desperate for sex as the night progressed - thus "all the girls get prettier at closing time." On the other hand, the measurements in the present study were all taken within a limited time frame of 9 p.m. to 12 a.m., the period during which young Australian adult pub and party socializing typically gets under way and well before closing time at the pubs ( 2 a.m.) or the end of the campus parties (more like 4 a.m.). Hence this interpretation does not appear very likely to be correct - although it cannot be ruled out.

In conclusion, despite its limitations the present study improved on previous studies of the "beer goggles" phenomenon by directly measuring BAC across a range of intoxication levels and by testing participants of both genders in the naturalistic settings of a campus pub and campus parties. A positive relationship between alcohol consumption and attractiveness ratings was clearly found, though the present results do not allow determination of whether the relationship reflects pharmacological actions of alcohol, alcohol expectancy, or some combination thereof. Intoxicated participants all knew they were under the influence of alcohol and conceivably could have responded in accordance with their beliefs about how intoxicated persons are 
supposed to behave in social situations (Hull \& Bond, 1986). Unfortunately the balanced placebo design that has been used to distinguish between effects of alcohol expectancy and the pharmacological actions of alcohol is unlikely to be successfully implemented at the moderate to high BACs that may be required to induce "beer goggles" (Lyvers \& Maltzman, 1991). In the present study, however, the fact that both moderate $(M=.05 \%)$ and high $(M=.16 \%)$ BAC groups showed the same "beer goggles" effect is consistent with recent neurophysiological evidence that alcoholinduced dopamine release in the reward system is most pronounced at moderate BACs and does not increase further with higher BACs (Olive \& Griffin, 2008). Thus if BAC increases perceived attractiveness due to alcohol's dopamine-releasing action, the "beer goggles" effect should occur at moderate BACs but no further increases should be evident at high BACs. Whatever the explanation, the relationship between alcohol consumption and perceptions of attractiveness may have significant health implications given the previous evidence cited above that high attractiveness in a prospective sexual partner increases the intent to engage in risky sex. 


\section{References}

Agocha, V.B. \& Cooper, M.L. (1999). Risk perceptions and safer-sex intentions: Does a partner's physical attractiveness undermine the use of risk-relevant information? Personality and Social Psychology Bulletin, 25, 746-759.

Alcholizer Technology ( 2007). Operation Manual H1/ H2. Cleveland, QLD: Author.

Bixler, R.H. (1989). Diversity: A historical/comparative perspective. Behavioral and Brain Sciences, 12, 15-16.

Dawe, S., \& Loxton, N. J. (2004). The role of impulsivity in the development of substance use and eating disorders. Neuroscience and Biobehavioral Reviews, 28, 343-351.

Franken, I. H. A., Muris, P., Georgieva, I. (2006). Gray's model of personality and addiction. Addictive Behaviours, 31, 399-403.

Friedman, R.S., McCarthy, D.M., Forster, J., \& Denzler, M. (2005). Automatic effects of alcohol cues on sexual attraction. Addiction, 100, 672-681.

Gladue, B.A. \& Delaney, H. J. (1990). Gender differences in perception of attractiveness of men and women in bars. Personality and Social Psychology Bulletin, 16, 378-391.

Hull, J.G., \& Bond, C.F. (1986). Social and behavioral consequences of alcohol consumption and expectancy: A meta-analysis. Psychological Bulletin, 99, 347-360.

Jones, B.T., Jones, B.C., Thomas, A.P., \& Piper, J. (2003). Alcohol consumption increases attractiveness ratings of opposite-sex faces: A third route to risky sex. Addiction, 98, 1069-1075.

Kruse, M. I., \& Fromme, K. (2005). Influence of physical attractiveness and alcohol on men's perceptions of potential sexual partners and sexual behavior intentions. Experimental and Clinical Psychopharmacology, 13, 146- 156. 
Lyvers, M., \& Maltzman, I. (1991). The balanced placebo design: Effects of alcohol and beverage instructions cannot be independently assessed. International Journal of the Addictions, 26, 963-972.

Olive, M.F., \& Griffin, W.C. Ethanol increases extracellular glutamate levels in the ventral tegmental area via D1 receptor activation. Presented at the Annual Scientific Meeting of the College on Problems of Drug Dependence, June 14-19, San Juan Puerto Rico.

Pennebaker, J. (1979). Don't the girls get prettier at closing time: A country and western application to psychology. Personality and Social Psychology Bulletin, 5, 122-125.

Putnam, S.K., Du, J., \& Hull, E.M. (2001). Testosterone restoration of copulation and medial preoptic dopamine release in castrated male rats: 2-, 5-, and 10-day treatments. Hormones and Behavior, 39, 216-224.

Rosen, R.C., \& Ashton, A.K. Prosexual drugs: Empirical status of the new aphrodisics. Archives of Sexual Behavior, 22, 521-543. 